\title{
THE RADIATIVE IMPACT OF FU ORIONIS OUTBURSTS ON PROTOSTELLAR ENVELOPES
}

\author{
K. R. BELL AND K. M. CHICK \\ Space Sciences Division, NASA Ames Research Center \\ MS 245-3 Moffett Field, CA 94035, USA \\ bell@cosmic.arc.nasa.gov, chick@anarchy.arc.nasa.gov
}

\begin{abstract}
We present preliminary results of an investigation into the radiative impact of FU Orionis outbursts on protostellar envelopes. In the thermal accretion disk instability model, the inner portion of the disk is inflated in such a way as to funnel most of the outburst luminosity along the poles of the system. A multidimensional radiative transfer code is employed to derive the thermal equilibrium structure of the surrounding protostellar envelope and the backheated accretion disk during outburst. The radiation emitted during outburst is modeled as a combination of a self-luminous accretion disk and a central point source. We compare the relative heating of circumstellar material due to (1) an isotropic point source and (2) an anisotropic point source in which the emitted radiation is confined to a $30^{\circ}$ cone about the polar axis. The isotropic point source creates a spherical dust cavity, while the anisotropic source naturally results in a cavity which is hourglass-shaped. Repeated outbursts may ultimately be responsible for the large-scale polar cavities commonly inferred to exist around young stellar objects. We also show that due to the anisotropy of the radiation expected during outburst, disk annuli within a few $a u$ will be shielded from the radiation of the outburst.
\end{abstract}

\section{Introduction}

Based on evidence suggesting that the driving sources of Herbig Haro $(\mathrm{HH})$ objects are transient but repetitive and that FU Ori outbursts release energy comparable to what is required to power observed HH outflows, it has been suggested that FU Orionis outbursts may be integral to the mechanism which gives rise to $\mathrm{HH}$ objects (Reipurth 1989). Several heavily embedded 
FU Ori systems which are associated with HH objects include L1551 IRS5 (in which $\mathrm{HH}$ objects are spaced at several hundred year intervals), HH57 (for which an $\mathrm{HH}$ object with a dynamical age of about $1000 \mathrm{yr}$ was discovered before the FU Ori system brightened and was identified), and Z CMa. None of the three "classical" FU Ori systems (FU Ori itself, V1057 Cyg, and V1515 Cyg) are known to have HH objects, but this may be due to a combination of their relatively advanced ages (inferred from small far IR excesses) and the fact that they are all viewed close to pole-on (Kenyon, Hartmann, \& Hewett 1988). Recent observations show that in several systems HH ejecta are dynamically clustered at periodic intervals with timescales similar to the expected FU Orionis outburst recurrence rate (HH34: 900 yr, Bally \& Devine 1994; HH200: 500 yr, Bally et al. 1995).

During FU Orionis outbursts, system luminosities increase by a factor of a hundred in a time as short as a few years; outbursts typically last several decades (Herbig 1977). Outbursts are thought to occur repeatedly during the first few hundred thousand years to all low mass, young stellar objects. Statistical arguments suggest that spacing between outbursts is between one and ten thousand years. Numerical calculations (Kawazoe \& Mineshige 1994; Bell \& Lin 1994) and the observations referred to above favor the smaller number. We refer to Hartmann \& Kenyon (1996) for a comprehensive review of the FU Ori phenomenon. In recent years, a detailed theoretical picture of FU Ori outbursts has emerged, in which a flare-up occurs in the central regions of an accretion disk (Lin \& Papaloizou 1985; Clarke, Lin \& Pringle 1990; Bell \& Lin 1994). This modeling suggests that during the outburst, the central disk becomes distorted in such a way that energy is strongly focused upward. In this paper, we apply this theoretical picture to study the effects of an FU Ori outburst on the protostar's placental environment.

One of the on-going puzzles in the field of low mass star formation is the origin of the polar cavities which seem to be ubiquitous among low mass, young stellar objects (Kenyon, Calvet, \& Hartmann 1993). Strong winds are common features of these systems, but it is not clear that the gas flow is capable of excavating a cavity. In particular, evidence indicates a high degree of collimation extending down close to the source of these outflows, yet the inferred cavities appear to have wide opening angles (Hartmann, Calvet, \& Boss 1996). Although collapse from spherical cores (eg. Cassen \& Moosman 1981) may not naturally result in optically thin lines of sight to the central object, systems which collapse from flattened sheets may result in the necessary geometry (Hartmann, Calvet, \& Boss 1996). As an alternative, we investigate the possibility that dust-free polar cavities may be the result of dust vaporization by irradiation during FU Orionis outbursts. 
Using a radiative transfer code with an assumed density distribution, we present preliminary results into an investigation of the thermal structure of the envelope during an FU Orionis outburst. In $\S 2$, we review the FU Orionis mechanism and summarize evidence which suggests that outburst luminosity will be emitted principally along the poles of the system. We also argue that FU Ori systems are deeply embedded objects likely to be surrounded by significant envelope material. We discuss our methods in $\S 3$. In $\S 4$, we show that the anisotropy of radiation emitted during outburst affects the thermal structure of the envelope and leads to the formation of bipolar, conical cavities. Source directionality will also reduce the irradiation of the surrounding accretion disk and may affect deduced bolometric luminosities. The current results are discussed in $\S 5$, and our plans for future work are given in $\S 6$.

\section{FU Orionis Outbursts}

Mass spirals inward through the accretion disk and lands on the central star at a rate which, although slowly declining with time, can be approximated as a radial constant at a given time. Mass transport is accompanied by the local generation of energy via viscous dissipation (Shakura and Sunyaev 1973). When mass flux through the disk is high enough to ionize hydrogen in the inner disk, the system will be subject to a thermal instability caused by the strong dependence of the $H^{-}$opacity on the temperature $\left(\kappa \sim T^{10}\right)$. Numerical calculations (Bell \& Lin 1994) suggest that as long as mass is transported into the inner disk at a rate greater than $5 \times 10^{-7} M_{\odot} y r^{-1}$, the inner disk will alternate between long periods of low mass flux quiescence ( $1000 y r, \sim 10^{-8} M_{\odot} y r^{-1}$ ) and short periods of high mass flux outburst $\left(\sim 100 \mathrm{yr}, \sim 10^{-4} M_{\odot} y r^{-1}\right)$. Time between outbursts varies from $800 \mathrm{yr}$ at an input mass flux of $10^{-6} M_{\odot} y r^{-1}$ to $1200 y r$ at $10^{-5} M_{\odot} y r^{-1}$ for a value of viscous efficiency of $\alpha=10^{-4}$. For larger values of $\alpha$ this interval decreases accordingly. Outburst and quiescent mass fluxes depend only on the stellar mass and inner disk cut-off radius $\left(M_{*}=1 M_{\odot}, R_{*}=3 R_{\odot}\right.$; Bell \& Lin 1994).

Models suggest that for $1 M_{\odot}$ systems, only the inner $1 / 3 a u$ of the disk will be subject to thermal instability (for higher $M_{*}$ this radius is larger). Light curves and color evolution of modeled outbursts agree well with observations of FU Orionis systems (Bell et al. 1995). A time sequence of B-band images of a model fit to the light curve of V1515 Cyg is shown in Figure 1. The image is viewed at an angle of $30^{\circ}$ from pole-on and has a radial extent of $0.3 \mathrm{au}$. During outburst, the inner disk is depleted in surface density. The slow migration of matter which fills in this diminished region during quiescence can be seen in the first three frames. In 1968, 


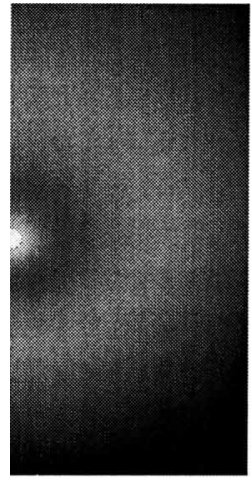

A.D. 1243

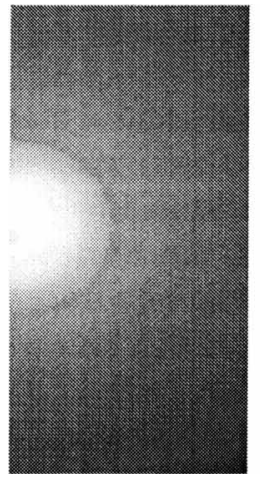

A.D. 1993

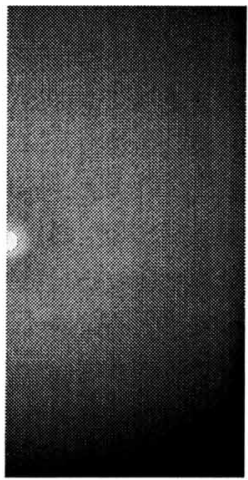

A.D. 1543

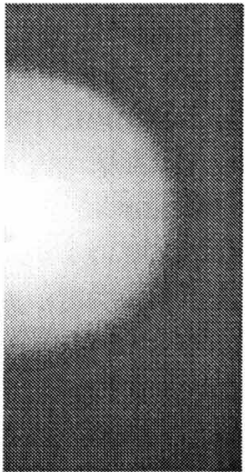

A.D. 2093

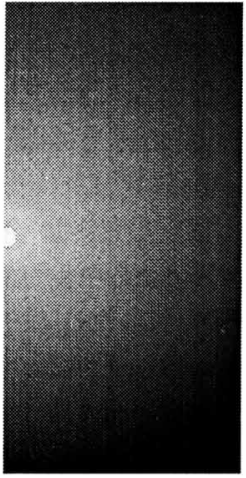

A.D. 1943

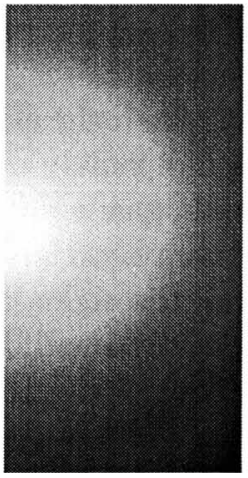

A.D. 2168

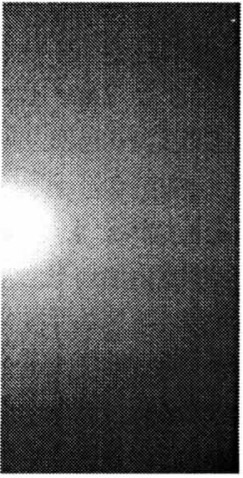

A.D. 1968

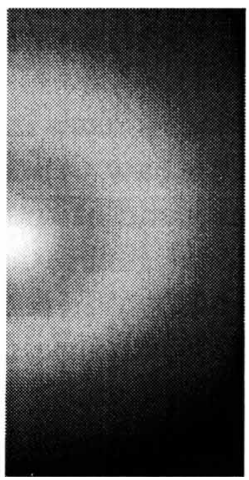

A.D. 2193

Figure 1. Progression of V1515 Cyg model B images throughout outburst cycle (from Turner, Bodenheimer, \& Bell 1997).

the brightening near the star signifies the recent onset of the outburst. The ionization front sweeps radially outward through the inner disk to a distance of about $1 / 4 \mathrm{au}$. The luminosity then fades uniformly through the inner disk and the system has returned to quiescence by 2193 .

Detailed simulations (Clarke , Lin, \& Pringle 1990; Bell \& Lin 1994; Bell et al. 1995) suggest that the inner disk surface during outburst is inflated inside the radius of hydrogen ionization; the brightest, hottest regions of the disk are consequently confined to a region within a few stellar radii which slopes strongly outward like the inside of a steep bowl. Radial cuts of three outbursting model disk shapes are shown in Figure 2. The labels $\mathrm{A} 1, \mathrm{~B} 1$, and $\mathrm{C} 1$ refer respectively to light curve fits of the three "classical" FU Ori systems: FU Ori, V1515 Cyg, and V1057 Cyg. For the B1 model 


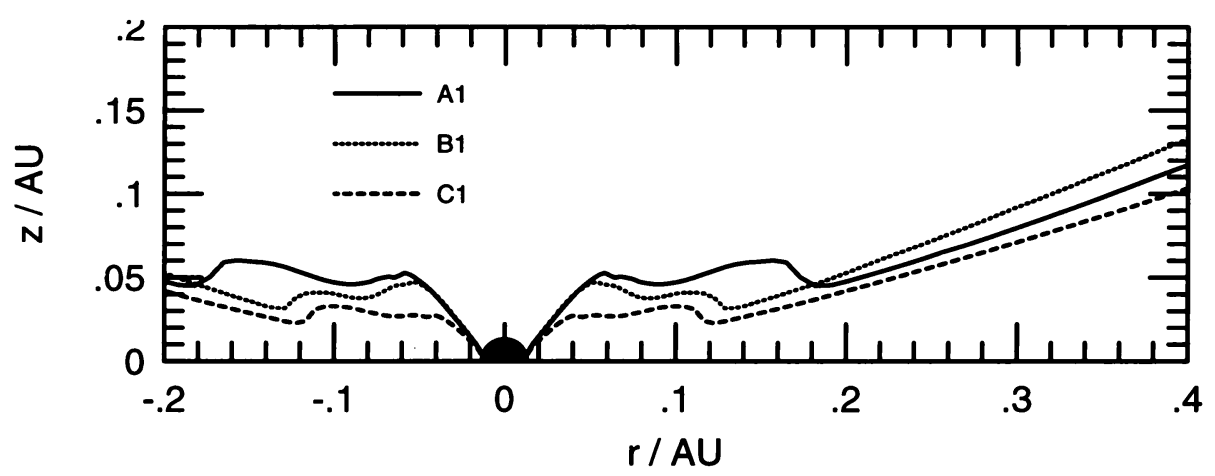

Figure 2. Shape of the inner disk during outburst with central star indicated (from Turner et al. 1997). The strongly sloping inner few stellar radii contain most of the luminosity of the system.

(the same that is shown here in Figure 1), the temperature at the peak in thickness at $.05 a u$ is only $4750 \mathrm{~K}$, down from its maximum of $7900 \mathrm{~K}$. The temperature continues to decrease rapidly with radius thereafter. Due to this geometry, we therefore expect that most of the radiation emitted during outburst will be focused upwards along the axis of the system.

Because of the high mass fluxes required to fuel the FU Ori cycle, it is expected that outbursts will be most common in the early evolution of a young stellar object when the remnant molecular cloud core is still dense. The existence of large scale circumstellar material is supported by images of nebulosity around most FU Ori systems (Herbig 1977, Goodrich 1987). Evidence for circumstellar material at much closer radius is given by observations of infrared emission in excess of disk continua (Kenyon \& Hartmann 1991). During decay from peak light of the V1057 Cyg outburst, emission from this excess component was seen to track the luminosity of the central object while radiation from intermediate wavelengths, due to emission by the constant mass flux outer disk, remained more nearly constant (Kenyon, Hartmann, \& Hewett 1988). Models derived from spectral energy distributions suggest that this material extends down to an $a u$ or so of the central object (Kenyon \& Hartmann 1991; Turner, Bodenheimer, $\&$ Bell 1997). Their very association with $\mathrm{HH}$ objects further supports the idea that FU Ori systems are enshrouded with circumstellar envelopes, because most $\mathrm{HH}$ objects are associated with the heavily embedded Class I or Class 0 protostars. 


\section{Method}

In this contribution, we present preliminary results of an investigation into the thermal impact of FU Ori outbursts on circumstellar envelopes. The radiative equilibrium structure of a model dust envelope is computed using an axisymmetric, multidimensional transfer code. The numerical scheme is summarized in the contributed poster by Chick \& Cassen (1997a), and described in detail by Chick (1997) and Chick \& Cassen (1997b). Scattering is not included in this procedure; for these relatively opaque models it is not expected to be a factor in the thermal balance. For the results presented here, the dust configuration is roughly toroidal and is based on theoretical models of collapse from a spherical cloud (Cassen \& Moosman 1981). The cloud is described by an initial rotation rate of $0.32 \times 10^{-14} \mathrm{sec}^{-1}$, a mass infall rate of $3 \times 10^{-6} M_{\odot} y r^{-1}$, and an age of $0.33 \times 10^{6} \mathrm{yr}$. The cloud infall geometry is characterized by a centrifugal radius of $1.1 \mathrm{au}$.

In the simulation, energy emanates from two sources: a central point source, and an infinitesimally thin, luminous accretion disk in the equatorial midplane. Energy emitted by the disk is specified by a standard model for accretion disks (Lynden-Bell \& Pringle 1974) with the same mass flux rate as the envelope: $3 \times 10^{-6} M_{\odot} y r^{-1}$. The temperature that the accretion disk surface reaches in radiative equilibrium with the warmed envelope is computed self-consistently. To test the effect of focusing emitted radiation along the axis likely to occur during outburst, heating by an isotropically emitting point source of luminosity $L$ is compared with heating by an anisotropic point source, in which the same luminosity is emitted into a cone of opening angle $30^{\circ}$. Given our choice of cloud and accretion parameters, it is consistent to assign the central source a luminosity of $L=28 L_{\odot}$ which is emitted at optical wavelengths. The disk has an intrinsic luminosity of $9 L_{\odot}$ which is emitted at thermal wavelengths.

\section{Results}

The results of the radiative transfer calculation are shown in Figure 3. The right panel shows the temperature structure for an isotropic central source and the left panel for one in which the same luminosity has been confined to a cone of $30^{\circ}$. Only one quadrant is calculated. The gray scale is linear temperature. The radial extent of each image is $3.8 \mathrm{au}$. Both envelope models have the same density distribution. The regions which are completely white are sufficiently hot such that dust grains have been destroyed and a cavity formed.

Regardless of the density distribution of the envelope, the size and shape of the dust cavity depends principally on the intensity and directionality of the central source. As shown in the right panel of Figure 3, a spheri- 

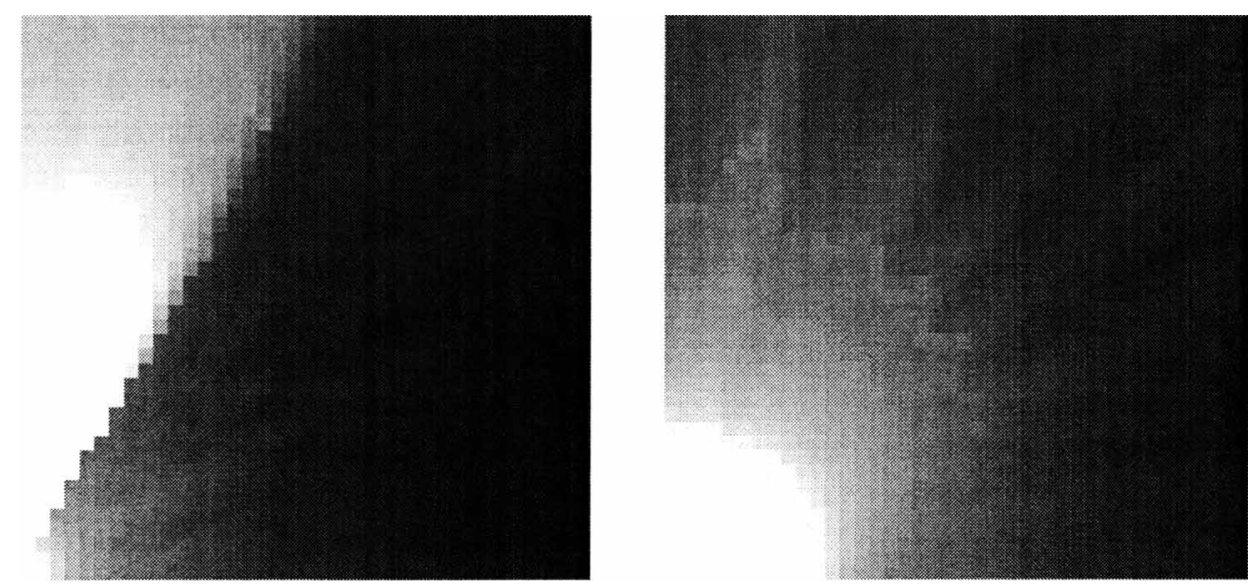

Figure 3. Temperature structure in envelope as a result of radiative transfer calculation. The non-isotropic source is on the left, the isotropic source is on the right. Both figures are plotted on the same linear scale; the lightest point is $1050 \mathrm{~K}$ and the darkest point is $350 \mathrm{~K}$. The white regions indicate the dust cavities where grains are vaporized. The scale of the plot is $3.8 \mathrm{au}$ on each side.

cal cavity is produced even in a highly flattened density distribution under illumination by an isotropic source (see also Chick, Pollack, \& Cassen 1996). Under illumination by the anisotropic source, however, a conical or hourglass-shaped dust cavity is created.

A further consequence of this anisotropic radiation field is the amount of heating experienced by disk material during outburst. The amount of radiation intercepted by the underlying accretion disk during FU Orionis outbursts may have implications for thermal processing of primitive materials in the solar nebula. The disk in this model has four potential heating sources: intrinsic viscous energy generation, central object radiation which has been captured by the envelope and reprocessed back down onto the disk, direct radiation from the central object, and radiation from other parts of the self-luminous disk. Because in this preliminary calculation, the disk is assumed to be infinitesimally thin, the last two sources are strictly zero.

The disk surface temperature given by the Lynden-Bell \& Pringle (1974) model is shown by the dashed line in Figure 4 . When radiation is emitted isotropically from the central point source, the envelope is heated uniformly down to the surface of the disk and the reradiation of energy onto the disk's surface is strong. The self-consistent disk temperature profile in this case is the top line in Figure 4. When central object radiation is beamed out the poles, temperatures are reduced globally throughout the cloud; the shape 


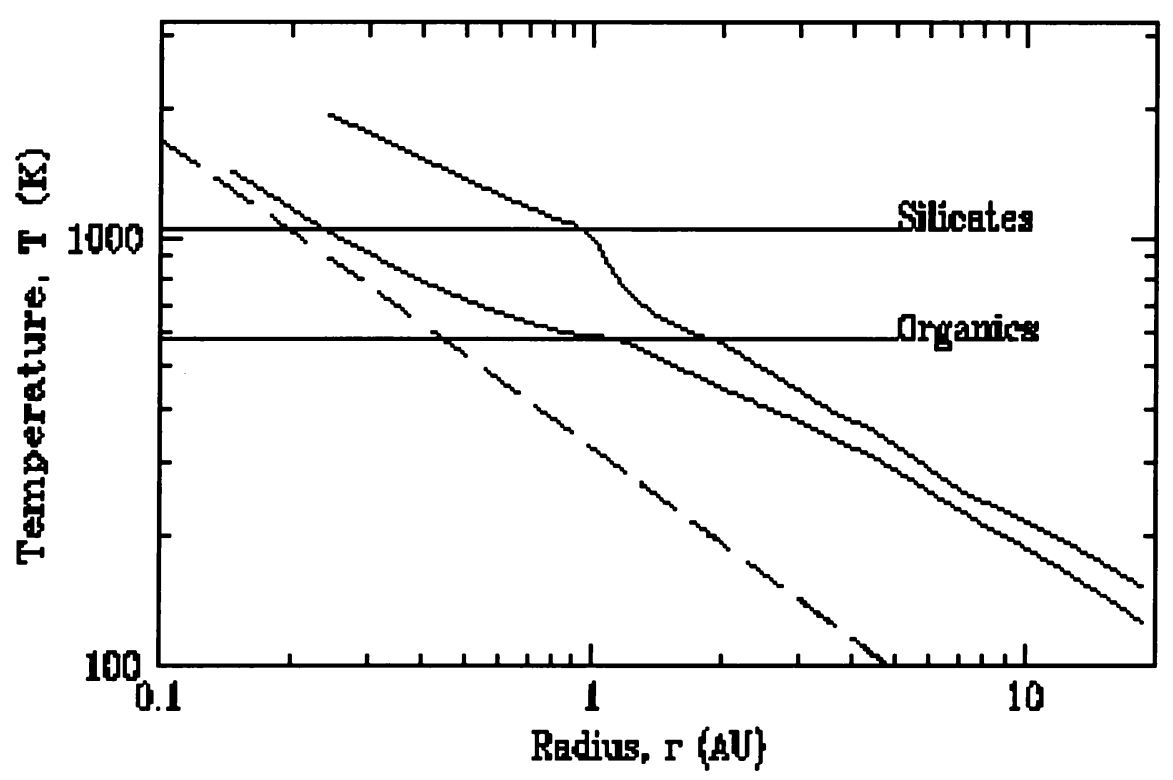

Figure 4. Radial temperature structure of the surface of the disk. The dashed line indicates the intrinsic disk profile due to locally generated accretion energy. The upper solid line includes the effects of radiation from the isotropic central source, the lower solid line from the non-isotropic source. The silicate and organic destruction temperatures are marked.

of the cavity and the disk temperature profile within a few $a u$ are considerably altered. There is a region where the disk is effectively shielded from the radiation of the central object and approaches the accretional surface temperature. Beyond a few $a u$, the thermal radiative transfer washes out the imprint of the anisotropic source. The envelope's temperature structure becomes more uniform, approaching spherical symmetry, and the slope of the disk's surface temperature profile parallels that of the isotropic source case at a somewhat lower value.

\section{Discussion}

Previous modeling of the impact of FU Ori outbursts on envelope and accretion disk has generally assumed isotropic radiation from the central source. Our modelling shows that far from the anisotropic source the thermal structure does become uniform and is qualitatively similar to the isotropic source case. (Although the thermal structure approaches uniformity, there will still be considerable differences in luminosity, effective temperature etc. be- 
tween equatorial and axial lines of sight.) Close to the center of the system, isotropic and anisotropic sources give rise to distinctly different thermal structures. Since radiation is beamed out the poles of the system, in the anisotropic case, temperatures are reduced globally throughout the envelope. Equatorial regions of the envelope and the accretion disk surface stay much cooler during outburst than with the isotropic central source. Our results suggest that within a few $A U$, source anisotropy will significantly reduce the heating by an FU Ori outburst of material in the solar nebula. The overall cooler envelope conditions may enhance the preservation of cometary ices. Due to dust vaporization, an hourglass-shaped cavity is opened along the cloud's axes. This is an efficient mechanism which may account for the ubiquitous polar openings inferred from observations of protostars and is independent of the details of infall physics.

In combination with the strong winds seen during FU Orionis outbursts, radiation emitted preferentially along the poles may help to power and enhance collimation of the jets and $\mathrm{HH}$ objects so commonly associated with these systems. Due to the increased heating of envelope material, the onset of outburst would create a sudden vaporization of dust particles. The front edge of new winds may therefore contain unusual and ionized species which may be detectable in some systems (as seen in Chandler \& Richer 1997).

The directionality of the FU Ori radiation may cause inaccuracies in estimates of bolometric luminosities. It is generally assumed that a young stellar object is an isotropically radiating system and that one need only integrate out to long enough wavelengths to measure all of its luminosity. Once a strong polar cavity is carved out of the envelope, however, some of the luminosity of the FU Ori outburst escapes entirely from the system along the poles (see also Chick, Pollack, \& Cassen 1996). This may account, for example, for the apparently anomalous low luminosity of edge-on system L1551.

\section{Future Work}

In this preliminary model, the disk has been assumed to be perfectly flat and to have no atmosphere. It is clear that the inner outbursting part of an FU Ori disk is quite complicated in structure. It has also recently been suggested that the outer constant mass flux part of the disk shows subtle behavior; in general the disk will be concave upward (and hence exposed to central object radiation) only out to the radius which marks the formation of dust grains $(\sim 1000 \mathrm{~K})$ and concave downward thereafter (Bell et al. 1997). Because photons travel at grazing angles over the surface of the disk, the details of the disk's atmosphere will also be taken into account to 
properly assess the true impact of FU Ori outbursts on the solar nebula's thermal structure. Rather than using an ad hoc directionally dependent point source as we do now, we will include the detailed structure of both inner and outer disk which results from the full numerical simulations to provide disk temperatures and thicknesses at each radius. We will also consider the effect of different envelope density structures.

Acknowledgements: We would like to thank Neal Turner for his efforts in producing a video of the outbursting models which was shown at the conference and for post script versions of the figures from his paper used in this contribution and in the oral presentation. K.R.B. would like to thank the conference organizing committee and Origins grant NAGW 4456 for funding her travel. K.M.C. acknowledges funding by the NASA Origins of Solar Systems program.

\section{References}

Bally, J. \& Devine, D. 1994, ApJ, 428, L65

Bally, J., Devine, D., Fesen, R. A., \& Laine, A. P. 1995, ApJ, 454, 345

Bell, K. R. \& Lin, D. N. C. 1994, ApJ, 427, 987

Bell, K. R., Lin, D. N. C., Hartmann, L. W., \& Kenyon, S. J. 1995, ApJ, 444, 376

Bell, K. R., Cassen, P. M., Klahr, H. H. \& Henning, Th. 1997 to appear September, ApJ, 486

Cassen, P., \& Moosman, A. 1981, Icarus, 48, 353

Chandler, C. J. \& Richer, J. S. 1997, in Low Mass Star Formation from Infall to Outflow: Poster Proceedings, Eds. F. Malbet \& A. Castets, p. 76

Chick, K. M. 1997, submitted to Journal of Quantitative Spectroscopy and Radiative Transfer

Chick, K. M., \& Cassen, P. 1997a, in Low Mass Star Formation from Infall to Outflow: Poster Proceedings, Eds. F. Malbet \& A. Castets, p. 207

Chick, K. M. \& Cassen, P. M. 1997b, ApJ, 477, 398

Chick, K. M., Pollack, J. B., \& Cassen, P. M. 1996, ApJ, 461, 956

Clarke, C. J., Lin, D. N. C., \& Pringle, J. E. 1990, MNRAS, 242, 439

Goodrich, R. W. 1987, PASP, 99, 116

Hartmann, L., Calvet, N., \& Boss, A. 1996, ApJ, 464, 387

Hartmann, L. \& Kenyon, S. J. 1996, ARAA, 34, 207

Herbig, G. H. 1977, ApJ, 217, 693

Kawazoe, E. \& Mineshige, S. 1994, PASJ, 45, 715

Kenyon, S. J., Calvet, N., \& Hartmann, L. W. 1993, ApJ, 414, 676

Kenyon, S. J. \& Hartmann, L. W. 1991, ApJ, 383, 664

Kenyon, S. J., Hartmann, L., \& Hewett, R. 1988, ApJ, 325, 231

Lin, D. N. C. \& Papaloizou, J. 1993, in Protostars and Planets III, eds. E. H. Levy \& J. Lunine (Tucson: Univ. of Arizona Press), 749

Lynden-Bell, D. \& Pringle, J. E. 1974, MNRAS, 168, 603

Reipurth, B. 1989, Nature, 340, 42

Shakura, N. I. \& Sunyaev, R. A. 1973, A\&A, 24, 337

Turner, N. J. J., Bodenheimer, P., \& Bell, K. R. 1997, ApJ, to appear May 